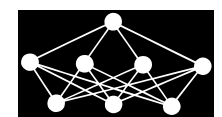

\title{
POSSIBILISTIC LVQ NEURAL NETWORK - AN APPLICATION TO CHILDHOOD AUTISM GRADING
}

\author{
C.S Kanimozhiselvi*, A. Pratap
}

\begin{abstract}
Psychological disorders are generally assessed by screening, behavioral features shown by the human. This challenges its accurate differential diagnosis and, is also error-prone when there are improper, insufficient clinical data and lack of clinical expertise. Thus, neural network based assistances in predicting and assessing psychological disorders have gained much interest. Childhood autism is a Neuro-psychiatric developmental disorder that impairs mainly three functional areas in a child: social, communication and behavior. This article demonstrates the application of a Possibilistic-Linear Vector Quantization 'Po-LVQ' neural network for the preliminary screening and grading of childhood autistic disorder. The diagnostic system assesses the grades as: 'Normal', 'Mild-Moderate', 'ModerateSevere', 'Severe'. It is able to perform with an improved overall accuracy of $95 \%$ exactly agreeing to the diagnostic criteria. Results of other performance parameters are also good enough to support the existing works about the applicability of neural network in autism diagnosis. Hence this research proposes a Po-LVQ based assessment support system for the diagnostic confirmation in grading childhood autism, during uncertain diagnosis due to lack of expertise. This helps to reduce the frustration and lengthy delays experiencing to parents before obtaining an accurate diagnostic result.
\end{abstract}

Key words: childhood autism, Possibilistic-linear vector quantization, artificial intelligence, artificial neural networks, assessment support system

Received: July 1, 2015

DOI: $10.14311 / N N W .2016 .26 .014$

Revised and accepted: March 20, 2016

\section{Introduction}

A computerized evidence based decision assist system enhances the quality and efficiency in clinical decision making through diagnostic assistances. These systems are designed to support or confirm clinical decisions rather than making the decisions for the user [5]. Many times a patient data is imprecise, uncertain and vague due to incomplete patient history or unnoticed symptoms. The theoretical

\footnotetext{
${ }^{*}$ Kanimozhiselvi C.S., Department of Computer Science and Engineering, KONGU Engineering College, Perundurai, Tamilnadu, India, Email: kanimozhi@kongu.ac.in

${ }^{\dagger}$ Anju Pratap - Corresponding author, Department of Computer Science and Engineering, SAINTGITS College of Engineering, Kottayam, Kerala, India, Email: anjuprathap@gmail.com
} 
knowledge in the symptom disease relationship and a vague patient data increases uncertainty in the diagnostic process.

From the clinical expertise, a diagnostician reasons logically and infer correctly before deciding or confirming a disease. A clinical diagnosis with lack of expertise will be inaccurate and uncertain. Thus the possibility of a misdiagnosis with either large volume or lack of functional data increases the difficulty in a correct diagnosis. Currently disease specific decision support systems are thus used to enhance the medical decision making procedure.

Artificial intelligence solves complex problems by simulating natural intelligence through reasoning from historical problems and their solutions. Machine learning is a growing discipline in the field of artificial intelligence, in which a computer machine can learn automatically based on a set of data and make decisions by recognizing complex patterns based on the data [1, 19]. Machine learning systems built using medical AI programs can help the healthcare workers in assisting tasks that depend on data and knowledge manipulation. Medical AI programs do different clinical tasks like diagnostic assistance. A diagnostic system can support or confirm a likely diagnosis by using the available patient data. Clinical assistance programs have the property of learning from case histories that are used for manual investigation. A medical diagnosis depends on the theory of anatomy, physiology, and also on the previous case histories and practical experience with other patients and its treatments. Thus, a machine learning model record diagnostic cases along with its salient features and solutions for building case bases to simulate human expertise. Then a similarity based mapping is applied between experience and the present situation.

The discipline of clinical psychology deals with mental health of human. The mental health data are generally uncertain by nature and the intensity of disorder may manifest differently in patients depending upon the degree of symptom present. Psychological disorders are mostly assessed by observing the qualitative behavioral features in a human. A clinical expertise is very important for the differential grading of a psychological disorder, which is comparatively difficult than diagnosing a disease. The computerized diagnostic assist system helps to solve any real world diagnosis problem through artificial reasoning, and have gained interest in clinical and nursing practices [6,9].

\subsection{Childhood autism}

Autism, which manifests in childhood, is a long term pervasive developmental disorder with persistent difficulties or disabilities in communication, social interaction and behavior, hence considered as a developmental disability or disorder. The prevalence of autism estimates that 1 in 150 children are affected by it and is three to four times likely to affect boys than girls. It shows that autism is one of the fastest growing psychological and developmental disorders in this world.

Even though childhood autism is not completely curable by medical treatments, early intervention makes a significant difference that increases the outcome. One of the facts about childhood autism is that, disability is not fully visible and the child looks like anybody without a physical disability, but with an inconsistent development. Moreover, autistic symptoms express itself in a wide variety with 
3 grades: Mild, Moderate and Severe. The invisible nature of the disorder with inconsistent development, make an accurate diagnosis harder and usually confirm the disorder on the basis of an assessment or second opinion. Children with autism can act differently with different grades. Hence, clinical expertise is very important for an accurate diagnosis and assessment for a prognosis of the disorder. In this real world, the process of clinical decision making with an inexperienced or fatigued clinician may have risks. The child's age has a direct relation with the behavioral features. An insufficient oxygen supply during birth increases the possibility of the disorder. However, its diagnosis immediately after birth is very difficult.

A questionnaire based categorization of the disorder has been practiced conventionally. A clinical psychologist collects the child's behavior through periodical direct observation and parental interview. These autistic behaviors are explained in assessment tools like Childhood Autism Rating Scale (CARS), Childhood Autism checklist for Toddlers (CHAT) etc. A tool calculates the total score attained by a child and grades the disorder accordingly. The grades range from Normal, MildModerate, Moderate-Severe and Severe. Generally, the total score is considered as an index to autism and to determine the requirement of remediation. Since the disorder mainly affects the effective communication and social behavior in a child, speech and psycho pathologists are involved in the non-medical (therapies) treatment procedures in order to alleviate the associated. Any defect or delays in the maturation of motor skills are likewise treated with the aid of a physiotherapist while, co-existing neurological problems like brain seizers should also be diagnosed and treated with medical procedures.

\subsection{Need of an assist system}

The accurate screening and assessing the severity of autism is a challenging task in the medical world. The onset of the disorder in very early age, its lack of awareness, un-noticed symptoms, its similarity with other neurological disorders etc. are the major factors that makes its diagnosis hard. Many high functioning autism cases go unnoticed in the society [24]. An early screening, assessment, right remediation and therapy sessions help the child to cope up with the social life fast. Therefore, screening and placing the disorder by an experienced clinician is highly needed for getting solutions for an affected child. But yet an expert clinician may experience uncertainty during an early diagnosis. Similarly, inappropriate screening and grading may result in 'diagnostic overshadowing' or an under-diagnosis [16].

In literature, there was a survey report about diagnosis over 1200 patients in London, and the parents expressed their practical challenges like lengthy delays before receiving a correct diagnosis and assessment [13]. In most cases, the diagnosticians go for another opinion for a diagnostic confirmation, the reason that makes the parents frustrating [10]. This lack of clinical expertise is an addressed problem. In addition to this, the symptomatic nature of autism also supports to the need of an assist system, comprises of experience and knowledge of a clinician, which can improve the strength of existing diagnostic procedure. It can confirm the assessment decisions of clinicians and can also utilize it for a diagnostic confirmation. 


\subsection{Artificial neural networks}

Artificial Neural Network (ANN) is an artificial intelligence based soft computing paradigm which models, both simple and complex systems by finding the invisible and non-linear relationships between input-output data. It is able to solve problems in prediction or forecasting, pattern recognition, classification or discrimination like human beings with acceptable accuracy. Hence it has gained much interest recently and widely applied to solve decision making problems. ANNs are structured like interconnected, weighted input-output units arranged in layers. It learns and stores the knowledge regarding a complex system directly from the data, by adjusting weights through a process of learning or training. Thus a learnt model is capable of generalizing unseen inputs, and forms relationship between inputs or between input and output, through learning from examples [8]. ANN Systems are tolerant and flexible towards missing, noisy and incomplete data. Moreover, they do not make any prior assumptions about data or attribute distribution and can be applied when the relationship between attributes are unknown [18]. ANNs are non-parametric and does not make any assumptions about the distributions of the associated data. It processes information in a complex non-linear fashion and are excellent for the typical types of data collected with rating scales. Likewise, the process of learning and generalization in ANN is analogous to a clinical decision making process where, clinicians learnt about a disorder by examining different cases and expresses expertise through diagnosing new cases[19]. Hence, these properties make them to widely apply in modeling, medical diagnosis problem, such that the models can support or assist doctors with their diagnosis by using the reported symptoms.

\subsubsection{Linear Vector Quantization neural network}

Learning Vector Quantization 'LVQ' is a prototype based supervised, competitive learning paradigm with data clustering technique in order to pre-process the dataset for obtaining cluster centers [18]. Data classification is established through a comparison on prototype vectors with labeled samples, which are seen during the preparation procedure. These prototypes represent typical data in the feature space and similarities between data are computed using similarity metrics. This similarity is too mentioned to as a quantization variation. Grounded on this similarity the closest prototype is updated, following the Winner-Takes-All (WTA) scheme. Then the production units are positioned and to approximate the decision surfaces. The net classifies an input by assigning it to the same division as the output unit that throws its weight vector closest to the input vector. Thus the LVQ network is having a layered architecture with two layers: a Competitive Layer and a Linear Layer. The competitive layer learns to classify the inputs by calculating the similarity between input vectors, to create subclasses. Then linear layer transforms these to user defined target classes. 


\section{Literature review}

There are recent studies on the application of artificial intelligence techniques in autism diagnosis. But researchers have taken up a different data collection and assessment methods for modeling the techniques, due to the unavailability of standard dataset. In psychological literature, the application of ANN in autism diagnosis has become a topic of interest $[3,18]$. The utility of neural networks in assisting the classification of autism has explored in early 1990's. A Feed Forward Back Propagation neural network that trained on 138 data, obtained from an autism assessment instrument called Autism Behavior interview was one among them [8]. In comparison with the traditional discriminant function analysis, neural networks performed superior with the classification ability on $92 \%$ vs. $85 \%$ basis during generalization testing. The criteria for autism diagnosis were also tested by building neural network models using 454 autism cases and 523 non-autistic cases. In a sample of 976 consecutive cases derived from the recent world-wide Field Trial of Autism and other Pervasive Developmental Disorders, we tested the accuracy of the 15 ICD-10 criteria for the diagnosis of Autism, by comparing neural network models $(\mathrm{NN})$ to more conventional multivariate competitors, namely, linear and quadratic discriminant function analysis and logistic regression. NNs were less accurate than competitors, both in terms of cross-validation results as well as in levels of shrinkage from training to test conditions [7]. The clinical research implications of these results are discussed [18].

Research has also aimed to maximize the cost effectiveness with easy to use models for supporting the clinical practitioners in diagnosing the disorder. For this, a fuzzy input based feed forward back propagation neural network was built using 40 samples; collected using a 6 items based checklist [3]. Thus, studies supported that neuro-fuzzy models would act as a supportive tool for medical practitioners with 85-90\% accuracy. But the implemented neuro-fuzzy model screens only the presence of the disorder, and could not be used for grading the disorder [18]. Progressively a hybrid artificial neural network model like DIAGADHD was also implemented using 165 samples for the diagnosis of Attention Deficit Hyperactive Disorder (ADHD) [4]. The results of a hybrid: Self Organizing Feature Map (SOM) neural network and Radial Basis Function (RBF) neural network have better accuracy than an individual back propagation algorithm [18].

The application of neural networks as an independent second opinion for autism diagnosis has been suggested in early 1990's [10]. A comparative study between Multilayer Perceptron (MLP) neural network and Logistic Regression technique strongly supported that neural networks are the best classifiers for this diagnostic problem [18]. The network was trained with 638 samples assessed using the Developmental Behavior Checklist (DBC). An accuracy of $80 \%$ was obtained during generalization testing.

Later, around 2006 another MLP was applied as a diagnostic assist in confirming the Learning Disability disorder [25]. A validation result of $89.74 \%$ for ANN shows that it can be used for diagnostic and screening tests. ANN classifiers like waveletchaos-neural networks are classified approximately $90 \%$ for processing EEG data and, for distinguishing patterns of speech and non - speech sound discrimination in autism $[2,18,26]$. 
There are studies and examples that support the ability of Fuzzy Cognitive Maps (FCM) in modeling complex systems for supporting decision making. Hence, researchers have applied the use of other artificial intelligence techniques like FCM for developing assist systems. It exploits the expertise by merging neural network and fuzzy logic methodologies. There was a research proposal based on the need for a methodology in assisting a speech therapist for the diagnosis of specific language impairments. An FCM model facilitated the differential diagnosis of Specific Language Impairment (SLI) from similar neurological disorders like autism and dyslexia [11]. The effectiveness of this FCM model was validated with four cases from the reported literature: two cases of SLI, a case of dyslexia and another case of autism. The experimental result agreed with the published results from the literature [18].

Certain classification results of FCM are overwhelmed by introducing non-linear Hebbian learning in the FCM. A set of 40 samples assessed using the Modified Checklist for Autism in Toddlers (MCHAT) was used for training an FCM Nonlinear Hebbian Learning (NHL) [14]. The model assisted with an average classification accuracy of $79.9 \%$, proposing its use as a guide in determining and planning therapies for autistic children. The performance of the same proposed FCM has again enhanced using FCM ensembles designed by using bagging and boosting methods [17]. The classification results support that the ensemble approach has a stable learning than a single non-linear Hebbian learning method.

Other machine learning techniques like clustering and classification have also applied for identifying the sub-types of Autism Spectrum Disorder [12]. Based on Autism Research Institute's (ARI) E2 survey consisting of 150 questions, samples are preprocessed using clustering algorithms like Expectation Maximization and Minimum Message Length algorithms [18]. The resulting clusters are classified using a JRip classification algorithm. Research studies have shown that associative classification mining techniques can also be applied to find the behavior pattern association in order to separate the autism disorder from Pervasive Developmental Disorder [22]. A dataset of 140 records collected by using a checklist for Behavior Test Assessment containing 32 criteria are pre-processed, and classification based on association has applied to predict accurate classes. The diagnostic results supported that Classification Based Association can be used for developing automated tools to assist clinicians in the diagnosis process.

An internet based expert system framework for the screening of autistic children has also proposed. The tool is named as a Delay Screener (DS) for evaluating developmental delays in a child [24]. This knowledge based screener not only helps to detect pervasive and socially developmental disorders, but also gives valuable training for children. Another expert system named PCADEX [21]-Pakistan Childhood Autism Diagnostic Expert System, which diagnosis autism based on autistic symptoms has also been developed. Its inference engine reasons from these rules and facts depending on the user's input, for making an optimum diagnostic decision. The rules are designed using the Autism Treatment Evaluation Checklist (ATEC) assessment instrument, a questionnaire containing 77 items. This system screens the presence of autism and classifies the level of the subject if autism present. 


\section{Data collection and preparation}

The collection of primary clinical data was somewhat challenging due to the social stigma and the parent's hesitation in recording the assessment data. This is the primary reason for the unavailability of any benchmark dataset. Hence the previous researchers have also collected autism assessment data available to their locality. In the first phase of data collection, knowledge is gathered by screening the common symptoms in childhood autism, group elicitation with domain experts and direct observation of autistic children from clinics. Childhood Autism Rating Scale (CARS) based psychological data is collected for study and implementation. CARS is one of the best validated instrument for diagnosis of childhood autism [21]. It is made up of 15 scales indicating various autistic features as shown in Tab. I [18].

\begin{tabular}{ccc}
\hline Sl. No & Descriptor & Symptoms of childhood autism \\
\hline 1 & RP & Impairment to Human Relations \\
2 & IM & Imitation \\
3 & ER & Inappropriate Affect \\
4 & BU & Bizarre Use of body movements \\
5 & OU & Peculiarities in relating to Non-Human Objects \\
6 & AC & Resistance to environmental change \\
7 & VR & Peculiarities in visual responsiveness \\
8 & LR & Peculiarities in auditory responsiveness \\
9 & TR & Near Receptive Responsiveness \\
10 & FR & Anxiety reaction \\
11 & VC & Verbal Communication \\
12 & NC & Non-verbal Communication \\
13 & AL & Activity Level \\
14 & IL & Intellectual Functioning \\
15 & GN & General Impressions \\
\hline
\end{tabular}

Tab. I $C A R S$ description.

Each descriptor is scaled with four items according to its level of severity and a scoring is used that ranges from Normal to Severely Abnormal as:

- 1 - Normal, shows that the child had never shown a problem,

- 2 - Mildly Abnormal, which means minimally impaired,

- 3 - Moderately Abnormal, which shows the child has significant impairment,

- 4 - Severely abnormal, which means a skill is almost nil.

- Midpoint scores like 1.5, 2.5 and 3.5 can be given if the feature appears in between the adjacent scores. The total score is calculated by adding up the individual scores of 15 items and, is classified in certain predetermined categories that represent the severity of autism. The total score possibly be ranged between 15 and 60 [18]. 
In the second stage, secondary CARS data have been collected for building the dataset. Thus, assessment data on 114 real world samples are collected from different clinics where 100 of them are collected initially, to build and test the applicability of the proposed technique. Each case record is labeled as: Normal, Mild-Moderate, Moderate-Severe and Severe, and the grade distribution is as represented in the Fig. 1 [18].

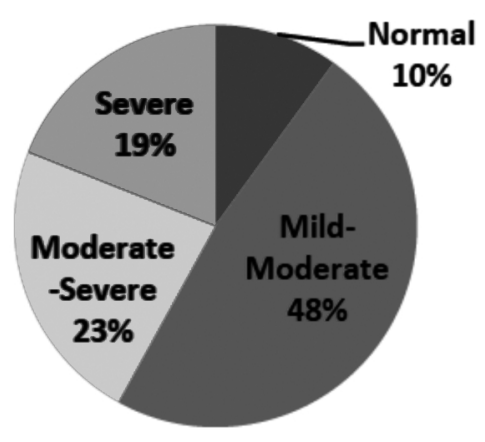

Fig. 1 Distribution of data in dataset.

Finally 14 new samples were collected later from a different clinic in order to test the generalization ability.

\subsection{Preparation of input to the neurons}

The assessment data is prepared and stored in Microsoft excel format for modeling the proposed method. The instances and descriptors are represented in rows and columns respectively. All identifying information is removed for protecting the children's and clinic's anonymity. The descriptor values are checked for valid ranges and invalid data are treated as missing attributes. Then missing data are identified and replaced with average values.

Thus the schema of the dataset was as a $100 \times 17$ matrix: 100 records, 16 descriptors and 1 autistic grade. Some statistical properties of the original data set are shown in Tab I.I in appendix [18]. Each record is diagnosed either as: Normal, Mild-Moderate, Moderate-Severe or Severe. There data distribution in the collected set is: 10 Normal cases, 48 Mild-Moderate, 23 Moderate-Severe and 19 Severe autistic cases.

\section{The possibilistic LVQ model}

The Possibilistic LVQ, otherwise represented as Po-LVQ, outputs the possibility of all the classes rather than giving a crisp output as the general LVQ neural network gives. By considering the possibility of adjacent classes, Po-LVQ is able to diagnose with better accuracy than general LVQ. For example, an input case with a total score of 30 is considered as 'Mild-Moderate' as per the diagnostic criteria. But the Po-LVQ calculates the possibilities of that case being 'Normal', 'Mild-Moderate' 
and 'Moderate-Severe', and then supports the class having maximum possibility. Suppose the next highest possible class is 'Normal', then it shows that the case will be able to improve after therapy sessions or it can be a false positive. Instead, if the next highest possible class is 'Moderate-Severe' then the strength of symptoms strongly supports the degree of severity of the disorder even though the total score is in borderline Mild.

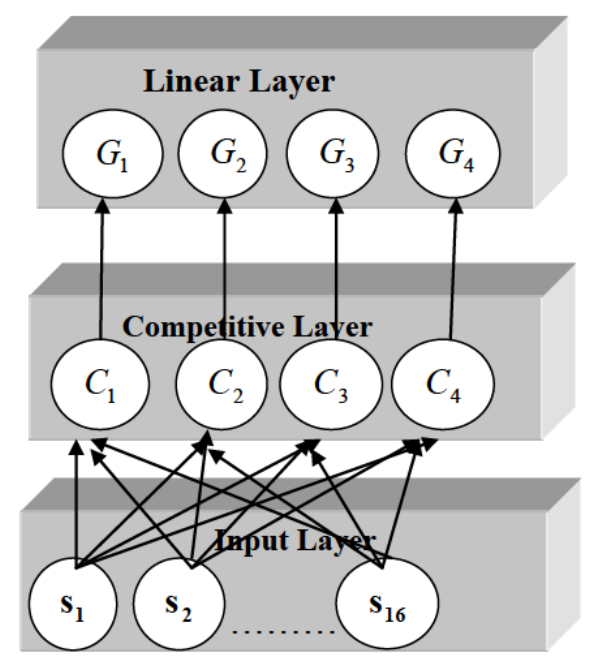

Fig. 2 Design of $L V Q$.

The design of the LVQ specific to the problem is as shown in Fig. 2 [18]. The input layer consists of 16 input neurons, which takes the 15 CARS descriptor values along with its total score, of a case record.

These 16 neurons represent the reception of an input vector $\mathbf{s}$ where $\mathbf{s}_{\mathbf{i}}$ is the score of $i$-th descriptor. The competitive layer learns the correlation between $\mathbf{s}$ and assigns them to $C$ prototypes in that stratum. Then, the linear layer transforms the prototypes into user defined classes or grades ' $G$ ' [18]. The process flowchart of Po-LVQ is shown in Fig. 3.

The possibility or belief of each neuron is computed by calculating the distance of the training sample $\mathbf{s}_{\mathbf{i}}$ from each neuron $C_{j}$ in the competitive layer, and then the maximum possibility neuron is declared as the winner. See Algorithm 1 for belief calculation [20]. The winning neuron moves towards the training sample in the rate of $+\alpha$ if it is in the same class of the input, else repulses from the training sample in the rate of $-\alpha$. Hence the symptom space is split to $C$ subclasses which in turn are connected, to form $G$ grades according to the target label in the training sample [18]. A codebook is constructed for each class using the symptom vector 's' of case histories. While diagnosing, the ' $m$ ' possibility of a test record with the set of codebook is calculated. The codebook that provides the highest possibility is considered as the grade on the test record. 


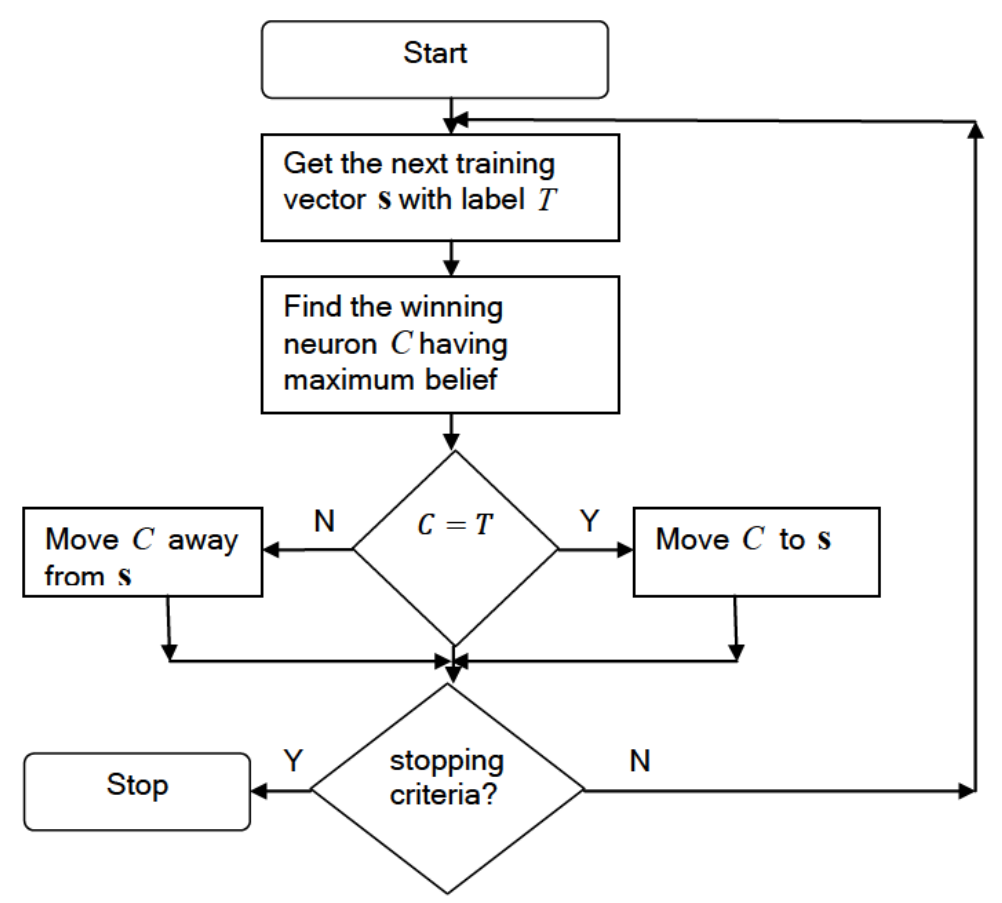

Fig. 3 Process Flowchart of Po-LVQ.

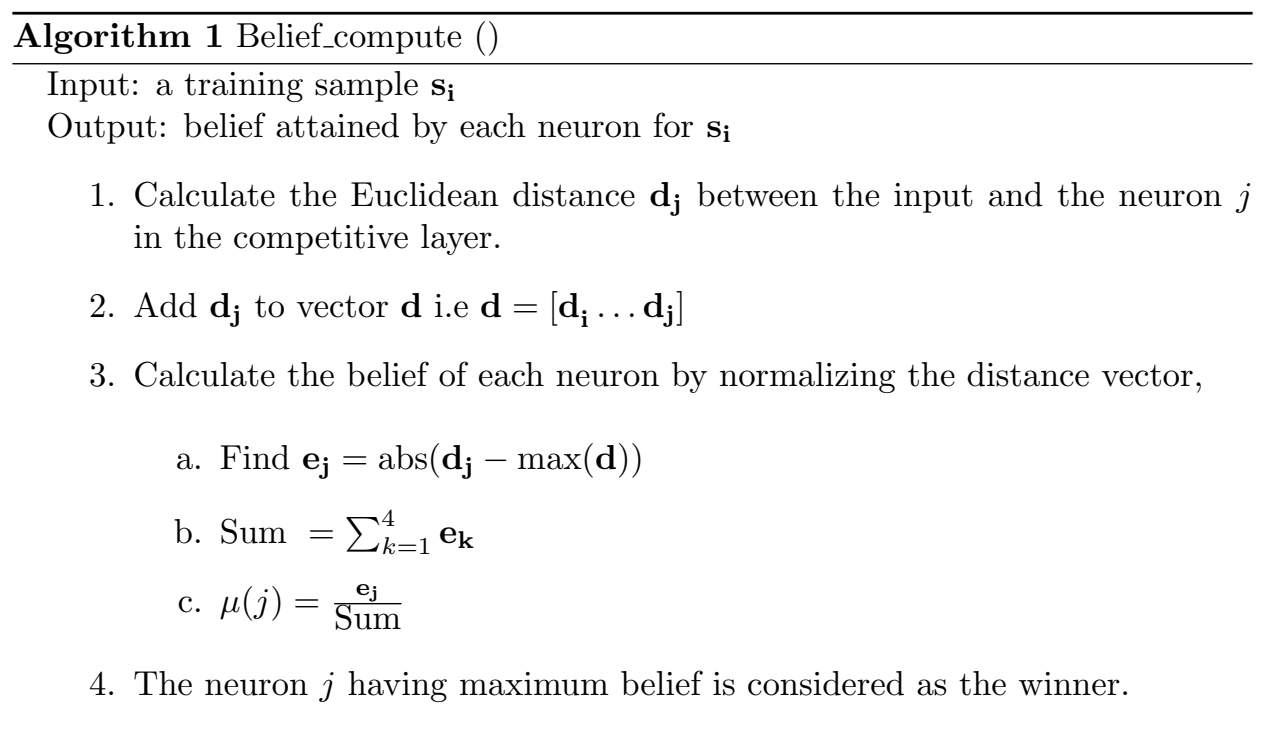

\section{Validation and evaluation}

The process of estimating performance, generalization ability and effectiveness of a model is referred to as its validation and testing. The Po-LVQ is validated using 
both internal and external methods. Internal validation uses a copy of the training samples for testing, where external validation considers only new testing samples. Fundamental evaluation metrics of a classifier are formulated from a table or matrix called confusion matrix. A confusion matrix is a performance vector with ground truth as rows and classification results as columns.

\subsection{Evaluation metrics used}

The selected 10 evaluation metrics [18] for assessing the performance of the proposed Po-LVQ is as follows:

1. Overall Accuracy is the total number of correctly graded records divided by the total number of test records, which shows the effectiveness of the classifier used.

2. Average Accuracy-It is the fraction of correctly classified records in each grade divided by the total number of records in each grade.

3. Average Reliability - It is the fraction of correctly classified records with regard to all records graded, as this grade in the test data.

4. Sensitivity is a measure of accuracy of autistic cases in the data set, i.e. how many of autistic cases are graded correctly. To multiclass problem, it refers to average sensitivity.

5. Specificity is a measure of accuracy of Normal (Non-autistic) cases in the dataset, i.e. how many of Normal cases are graded correctly

6. Kappa statistic estimates the strength of agreement in diagnosis made between a clinician and a classifier.

7. Geometric Mean (G-Means) is a metric that indicates the balance between classification performances on the majority and minority classes.

8. Likelihood Ratio for a negative test result, LR (-) is the ratio between the probability of predicting an example, as negative when it is actually positive, and the probability to predict an example as negative when it is truly negative.

9. Balanced Accuracy is the average of Sensitivity and Specificity and, can be defined as the average accuracy obtained on either class.

10. Youden's index $\gamma$ evaluates the algorithm's ability to avoid failure [27].

\subsection{Observations}

Re-substitution testing is a widely accepted and simple internal validation method when there is a shortage of real world data for estimating the overall accuracy. The Po-LVQ model diagnoses an input data as one among the four labels as discussed in the previous section. The confusion matrix for this 4-class problem is represented as $4 \times 4$ square matrix, as indicated in the Tab. II. 
Neural Network World 3/2016, 253-269

\begin{tabular}{ccccc}
\hline Grades & Normal & Mild-Moderate & Moderate-Severe & Severe \\
\hline Normal & 10 & - & - & - \\
Mild-Moderate & - & 46 & 2 & 0 \\
Moderate-Severe & - & - & 22 & 1 \\
Severe & - & - & 2 & 17 \\
\hline
\end{tabular}

Tab. II Confusion matrix obtained.

It is noted that all 10 records out of 10 are classified as Normal, 46 out of 48 is classified as Mild-Moderate and 2 as Moderate-Severe, 22 out of 23 is classified as Moderate-Severe where 1 as Severe and 2 out of 19 is classified as Moderate-Severe and 17 as Severe itself. As Po-LVQ uses class labels for its prototype- belief based learning; it is able to show an overall accuracy up to $95 \%$. The experimental and comparative results based the evaluation particulars at a learning rate of 0.8 are as shown in Tab. III [18].

\subsubsection{Similarity in assessment}

Fig. 4 represents the similarity between the grading of Po-LVQ and the CARS tool. The $\mathrm{x}$ and $\mathrm{y}$ axes represent the number of case records and the autism grades respectively.

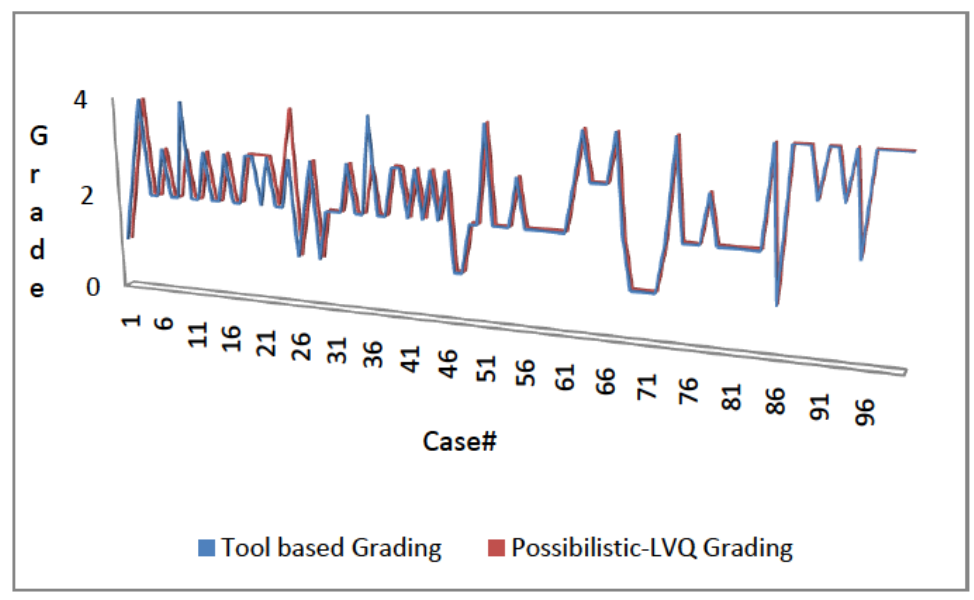

Fig. 4 Grading comparison of the proposed model with CARS tool.

\subsubsection{Case studies for testing generalization ability}

The generalization ability and effectiveness of the proposed model are validated through external methods, by selecting 14 new case histories from a Homoeo institute in Kerala, India. The data distributions were: 10 severe, 2 Mild-Moderate 
and 2 Moderate-Severe [18]. Diagnostic results show cent percent agreement with CARS diagnostic criteria. The Po-LVQ clearly graded the three inputs as per the diagnostic criteria, supporting its generalization ability.

\subsubsection{Balanced dataset}

A phenomenon in which classes in a dataset are not distributed uniformly is referred as class imbalance. One class may have much less training data than remaining classes. This may occur in real world problems where there is a lack in data availability like rare diseases.

But learning from an imbalanced dataset may degrade the classification accuracy [18]. Hence the performance of Po-LVQ is confirmed with a balanced dataset prepared using a random under sampling method. This keeps all minority classes while extracting smaller set of redundant majority classes. A manual random under sampling is performed for balancing the dataset by selecting 44 samples uniformly. But the model classified nearly 43 samples out of 44 correctly, thereby achieving a consistent overall accuracy of 97.72 .

\subsection{Result analysis and discussion}

It is noticed that, Po-LVQ shows an improvement to general LVQ with respect to the performance particulars. Fig. 5 represents a graphical comparison of the above two. The result comparison is tabulated in Tab. III.

\begin{tabular}{ccc}
\hline Particulars & Po-LVQ & LVQ \\
\hline Average Accuracy & 0.952397979 & 0.9369 \\
Average reliability & 0.947649573 & 0.948 \\
Sensitivity & 0.936530638 & 0.91 \\
Specificity & 1 & 1 \\
Kappa & 0.925948 & 0.911 \\
Gmeans & 0.967745131 & 0.957 \\
LR(-) & 0.063469362 & 0.084 \\
Bal_ACC & 0.968265319 & 0.958 \\
Youden Index & 0.936530638 & 0.916 \\
\hline
\end{tabular}

Tab. III Performance comparison.

A sensitivity of 0.936 suggests that Po-LVQ is good for the diagnostic assessment test which assesses the severity level of the disorder. Similarly, a specificity of 1 suggests that, it also good for diagnostic screening test that detects the presence or absence of the disorder. The obtained Kappa coefficient ' $\mathrm{K}$ ' is 0.9259 , which shows that the strength of agreement is very good for the records that are independently diagnosed using assessment tools and Po-LVQ. For each grade of ground truth records, on an average $94.7 \%$ of grades are graded reliably with regard to all grades in the test data. The above observations recommend the application of a Po-LVQ trained with the collected dataset, for grading childhood autistic disorder. Its 


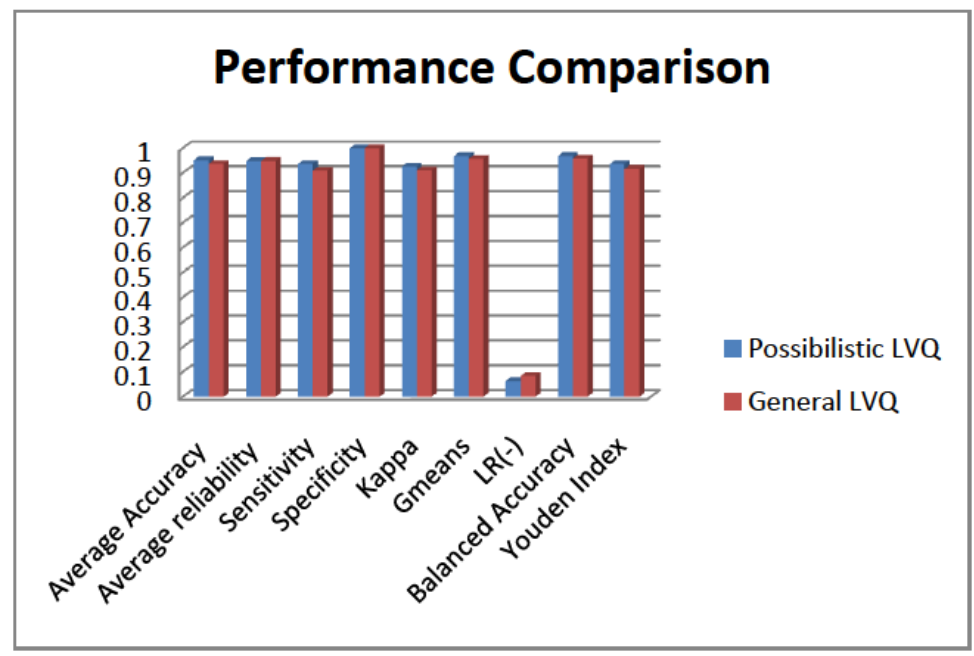

Fig. 5 Performance comparison: Po-LVQ vs. general $L V Q$.

performance suggests the usage while developing autism assessment systems for grading childhood autism.

The literature suggests the application of Feed Forward Neural Networks trained with backpropagation algorithm (MLP) for autism diagnosis and prediction. Lack of a benchmark dataset has made the use of different dataset in each literature. This research study also applied a MLP trained with collected samples, for a general comparison with LVQ with respect to accuracy [18]. The result is as shown in Tab. IV. MLP was validated using re-substitution cross validation and split validation methods. Training cycles were 100 and 500, which shows a better accuracy in 500 .

\begin{tabular}{ccccc}
\hline Grade & Actual data & LVQ & Po-LVQ & MLP \\
\hline N & 10 & 10 & 10 & 8 \\
MM & 48 & 46 & 46 & 45 \\
MS & 23 & 23 & 22 & 19 \\
S & 19 & 15 & 17 & 19 \\
Overall Accuracy & 100 & 94 & 95 & 91 \\
\hline
\end{tabular}

Tab. IV Po-LVQ vs. other models.

\section{Conclusion}

Artificial intelligence in medicine consists of, problem solving methods that simulate expertise and domain knowledge for diagnostic classification of diseases; by developing assist systems for clinicians that can be used during uncertainty or vagueness 
in medical data. Childhood autism is a Neuro-psychological disorder in which early intervention may alleviate the associated problems and gives a long term outcome. Autism assessment is traditionally done by assessment instruments, which classify and grades a person using a score based methodology. The application of medical artificial intelligence has become an application and area of research in the psychological field. Medical AI can be applied in diagnostic problems by simulating experience and embedding medical knowledge. This paper proposed a possibilistic $L V Q$ based screening and grading of childhood autism to four possible grades. The implementation results are compared with the performance of a general LVQ and other existing applied models. Based on the result comparison, Po-LVQ based childhood autism grading is seemed to be better and can be applied to grading childhood autism while developing an autism assessment support system.

\section{Acknowledgement}

We are thankful to the Clinical Psychologists: Dr. Aniamma Mathew and Ms. Anupama, from Kerala, India for giving relevant domain knowledge for testing.

\section{Appendix}

\begin{tabular}{ccccc}
\hline Property Name & 1 & 2 & 3 & 4 \\
\hline No. of cases & 10 & 48 & 23 & 19 \\
Standard deviation & 1.4757 & 1.078 & 0.7175 & 1.9945 \\
Minimum & 24.5 & 29 & 33 & 36 \\
Maximum & 28.5 & 32.5 & 35.5 & 43 \\
Mean & 26.7 & 30 & 33.913 & 38.684 \\
\hline
\end{tabular}

Tab. V Statistics of dataset.

\section{References}

[1] ALPAYDIN E. Introduction to machine learning. England. MIT press. 2004, doi: 10.1017/ S0269888910000056.

[2] ADELI A., NESHAT M. A fuzzy expert system for heart disease diagnosis. In: Proceedings of International Multi Conference of Engineers and Computer Scientists (IMECS 2010), Hong Kong, 2010.

[3] ARTHI K., TAMILARASI A. Prediction of autistic disorder using neuro fuzzy system by applying ANN technique. International journal of developmental neuroscience. 2008, 26(7), pp. 699-704, doi: 10.1016/j.ijdevneu.2008.07.013.

[4] ARTHI K., TAMILARASI A. A hybrid fuzzy model in prediction of ADHD using artificial neural networks. Journal of Neural Systems Theory and Applications. 2011, 1(1), pp. 1-17.

[5] CASTILlO R.S., KELEMEN A. Considerations for a successful clinical decision support system. Computers Informatics Nursing. 2013, 31(7), pp. 319-326, doi: 10.1097/nxn. Ob013e3182997a9c. 


\section{Neural Network World 3/2016, 253-269}

[6] CHO I., KIM J., KIM J.H., KIM H.Y., KIM, Y. Design and implementation of a standardsbased interoperable clinical decision support architecture in the context of the Korean HER International journal of medical informatics. 2010, 79(9), pp. 611-622, doi: 10.1016/j. ijmedinf.2010.06.002.

[7] CICCHETTI D.V., VOLKMAR F., KLIN A., SHOWALTER D. Diagnosing autism using ICD-10 criteria: A comparison of neural networks and standard multivariate procedure. Child Neuropsycology. 1995, 1(1), pp. 26-37, doi: 10.1080/09297049508401340.

[8] COHEN I.L., SUDHALTER V., LANDON-JIMENEZ D., Keogh M. A neural network approach to the classification of autism. Journal of autism and developmental disorders. 1993, 23(3), pp. 443-466, doi: 10.1007/bf01046050.

[9] DOWDing D., MITCHELL N., RANDELL R., FOSTER R., LATTIMER V., THOMPSON, C. Nurses' use of computerised clinical decision support systems: a case site analysis. Journal of clinical nursing. 2009, 18(8), pp. 1159-1167, doi: 10.1111/j.1365-2702.2008. 02607.x.

[10] FLORIO T., EINFELD S., TONGE B., BRERETON A. Providing an independent second opinion for the diagnosis of autism using artificial intelligence over the internet [online]. Counseling, Psychotherapy and Health: The Use of Technology in Mental Health. 2009, 5(1) pp. 232-248 [viewed 2016-06-27]. Available from: https://www.mentalhealthacademy.net/ journal_archive/cph0812.pdf.

[11] GEORGOPOULOS V.C., MALANDRAKI G.A., STYLIOS C.D. A fuzzy cognitive map approach to differential diagnosis of specific language impairment. Artificial intelligence in Medicine. 2003, 29(3), pp. 261-278, doi: 10.1016/S0933-3657(02)00076-3.

[12] GUILLÉN R., JENSEN C., EDELSON S. A machine learning approach for identifying subtypes of autism. In: Proceedings of the 1st ACM International Health Informatics Symposium. ACM, 2010, pp. 620-628, doi: 10.1145/1882992.1883091.

[13] HOWLIN P., MOORE A. Diagnosis in autism a survey of over 1200 patients in the UK Autism. 1997, 1(2), pp. 135-162, doi: 10.1177/1362361397012003.

[14] KANNAPPAN A., TAMILARASI A., PAPAGEORGIOU E.I. Analyzing the performance of fuzzy cognitive maps with non-linear hebbian learning algorithm in predicting autistic disorder. Expert Systems with Applications. 2011, 38(3), pp. 1282-1292, doi: 10.1016/j. eswa.2010.06.069.

[15] LEE S. Features of computerized clinical decision support systems supportive of nursing practice: a literature review. Computers Informatics Nursing. 2013, 31(10), pp. 477-495, doi: $10.1097 / 01 . n c n .0000432127 .99644 .25$.

[16] LEVITAN G.W., REISS S. Generality of diagnostic overshadowing across disciplines. Applied research in mental retardation 1983, 4(1), pp. 59-64, doi: 10.1016/S0270-3092(83)80018-6.

[17] PAPAGEORGIOU E.I., KANNAPPAN A. Fuzzy cognitive map ensemble learning paradigm to solve classification problems: Application to autism identification. Applied Soft Computing. 2012, 12(12), pp. 3798-3809, doi: 10.1016/j.asoc.2012.03.064.

[18] PRATAP A. A study on the application of soft computing techniques for grading childhood autism. Chennai, 2015. PhD thesis, Anna University Chennai, India.

[19] PRATAP A., KANIMOZHISELVI C.S. Predictive assessment of autism using unsupervised machine learning models. International Journal of Advanced Intelligence Paradigms. 2014 6(2), pp. 113-121, doi: 10.1504/IJAIP.2014.062174.

[20] PRATAP A., KANIMOZHISELVI C.S., VIJAYAKUMAR R., PRAMOD K.V. Parallel Neural Fuzzy-Based Joint Classifier Model for Grading Autistic Disorder. In: Soft Computing Applications, Proceedings of the 6th International Workshop Soft Computing Applications (SOFA 2014). Springer International Publishing, 2016, pp. 13-26, doi: 10.1007/ 978-3-319-18296-4_2.

[21] SAJJAD S., HIRA QAMAR K.T., BANO S. Development of a Diagnostic Expert System for Autism Disorder-PCADEX [online]. Department of Humanities COMSATS Institute of Information Technology, 2012. Islamabad, PAKISTAN [viewed 2016-06-27]. Available from: https://www.researchgate.net/publication/228520330. 
Kanimozhiselvi C.S., Pratap A.: Possibilistic LVQ neural network...

[22] SUNSIRIKUL S., ACHALAKUL T. Associative classification mining in the behavior study of Autism Spectrum Disorder. In: Proceedings of the second international conference on Computer and Automation Engineering (ICCAE), Singapore. IEEE, 2010, 3, pp. 279-283, doi: 10.1109/iccae.2010.5451851.

[23] SWIERCZ M., KOCHANOWICZ J., WEIGELE J., HURST R., LIEBESKIND D.S. MARIAK Z., MELHEM E.R., KREJZA J. Learning vector quantization neural networks improve accuracy of transcranial color-coded duplex sonography in detection of middle cerebral artery spasm-preliminary report. Neuroinformatics. 2008, 6(4), pp. 279-290, doi: $10.1007 / \mathrm{s} 12021-008-9023-0$.

[24] VEERARAGHAVAN S., SRINIVASAN K. Exploration of autism expert systems. In: Proceedings of 4th International Conference on Information Technology (ITNG'07), Las Vegas, Navada. IEEE, 2007, doi: 10.1109/ITNG.2007.91.

[25] WU T.K., MENG Y.R., HUANG S.C. Identifying and diagnosing students with learning disabilities using ANN and SVM. In: Proceedings of the 2006 IEEE International Joint Conference on Neural Networks (IJCNN'06), Vancouver, Canada. IEEE, 2006, pp. 4387-4394, doi: 10.1109/IJCNN. 2006.247038.

[26] YOU X., TENG N., AYALA M., WANG L., BARRETO A., RISHE N., ADJOUADI M A practical EEG study on autism using Artificial Neural Networks. In: Proceedings of the IASTED International Conference on Graphics and Visualization in Engineering, Florida, USA. ACTA Press, 2007, pp. 41-46.

[27] YOUDEN W.J. Index for rating diagnostic tests. Cancer. 1950, 3(1), pp. 32-35, doi: 10 1002/1097-0142(1950) 3:1\$<\$32: : aid-cncr2820030106\$>\$3.0.co; $2-3$. 